work can be further helped by making this course coincide, when possible, with the lectures on surgery.

The subjects to be taught and the order and manner in which they shall be approached are matters on which all may differ and still attain good results. I have found the old method followed in the teaching of surgery, according to systems, very satisfactory and see no reason to change. I usually begin by considering the fundamental essential for surgery, namely, regeneration and repair in the various character of tissues; then I take up the diseases of the skeletal system, the surgical affections of the gastrointestinal system from the lips to the anus, including the gall bladder and pancreas. the thyroid gland, the genital system in male and female, including the breast, the surgical affections of the urinary system and, finally, the lymph system and miscellaneous conditions, as skin tumors, etc.

In treating all these subjects, whenever time will permit, a very hurried survey is first made of the anatomy, histology and physiology of the organ or region to be studied in order that the student may have at hand all the data necessary for the proper application of his pathologic knowledge to the study of the living patient. The pathology of the condition and the latest experimental findings are then gone into as carefully as possible with especial stress on their relationship to other organs and the region affected when such relationship exists. Methods of transference of conditions with routes, whenever traceable, are studied in detail, as in the case of metastasis of tumors, etc. Symptoms and recognized methods of treatment are touched on only when there is a pathologic lesson to be derived or whri? their own origin is easily and distinctly traceable to some plainly demonstrable macroscopic or microscopic pathologic condition. In other words, the student's attention is called to symptoms only when necessary to show how clearly dependent they are on abnormalities of structure, causing abnormalities in physiologic function. He is not required to know them as forming a clinical entity, but whenever possible an effort is made to make it easy for him to conjecture as to their presence from a simple process of deductive reasoning. The whole subject is then clinched by a couple of hours spent in the laboratory studying microscopic and gross specimens covering the subject considered.

This I submit as my answer to the query, "What is surgical pathology?" The subject as a distinct specialty is comparatively young, and courses in the various colleges are of very recent date. We feel that with proper encouragement both by siudents and co-educators much good can be accomplished for the student, his burdens made lighter instead of increased, his mental attiture toward medical subjects broadened, and at the same time there will develop a set of men trained in experimental surgery and pathology, well equipped to take up the burdens when time shall rob us of the masters of to-day.

2131 North Fifteenth Street.

Carbohydrates in Diabetes.-I. V. Lemann, in the Nero Orleans Medical and Surgical Journal, April, states that each case demands separate study, because cases differ not only in grade but in kind also. The tolerance of patients to various carbohydrates is different. Various complications call for alterations in diet, and unless the physician is prepared to give intelligent attention to these details he can not hope for good results from the diet he prescribes for his diabetic patients.

\section{CANCER OF THE BRFAST}

\section{F. S. DENNIS, M.D.}

NEW YORK

In the present contribution, I shall refer only incidentally to statistics. In several previously published papers $^{1}$ I have given my statistics at length. I desire now to express some opinions that I have formed and facts that I have collected from a study of my own personal cases during the past twenty-five years, selecting a few cases of cancer of the breast, and analyzing them critically, because $I$ believe it is a better way to arrive at the truth, than by a promiscuous study of a large number of cases with statistics only as a guide to determine certain important facts.

I intend to consider very briefly some salient facts in regard to the final outcome of thirty-nine cases of cancer of the breast, the histories of which I know from the time of the operation to the present day. These thirtynine cases have been selected from a large number. solely for the reason that I have a personal knowledge of each case from the incipiency of the disease to the present moment. All these patients have lived bevond the three years' limit of time. One patient has lived twenty-five years, another nineteen years, another seventeen years, and another fifteen years, and the rest from three to fourteen years with no return of the disease. In this list one was eighty-five years and another eightytwo years old. I mention thesc two cases to illustrate the fact that age itself appears to offer no barrier to an operation for the reliet of cancer of the breast.

I am convinced that a study of the cure of cancer of the breast from a percentage point of view only has created a very false impression among surgeons and a depressing effect on the laity. The all-important question for the surgeon and the patient is this: Can a permanent cure be effected if the operation is undertaken early, and radical in character? If this view can be substantiated it will create hope in the patient, and inspire courage in the surgeon.

The clinical picture of cancer of the breast has been so drawn in past years as to create a most unfavorable impression. The early and radical operation is certain to efface this gloomy picture, and to draw in its place ane that will inspire hope in the unfortunate victims of the disease. The safety of the operation is a subject worthy of consideration. In all of the operations which I have mentioned, and an accurate knowledge of which I possess, there has not been a death except in two cases of hemophilia, and such deaths are not peculiar to this special operation, but would occur in connection with any other major operation. The mortality therefore, in several hundred cases would be almost nothing, even if these cases were included, and absolutely nothing if they were excluded. The percentage of permanent cures, on the other hand, can not be so correctly estimated because so many cases are lost sight of and therefore can not furnish data for statistical purposes. In a recent small series of cases extending over three years from the time of the operation the percentage of permanent cures amounted to $7 \%$ per cent. In a previous series the percentage was 45 per cent., but these operations were done earlier in the history of what is called the complete operation. The sum total of percentages of cure therefore. would fall below the high figure previously mentioned, and above the lower figure stated in the second series. These facts certainly en-

1. Tr. Am. Surg. Lnssn. 
coulage the surgeon to hope for even better results, and as we learn more about the disease and still further improve the technic of the present operation, the future holds out even greater encouragement for more brilliant results in the cure of a disease, which in former years baffled the skill of the greatest surgeons of the day.

\section{PERMANENT CURE}

The permanent cure of cancer of the breast is a subject in which the surgeon as well as the patient take the greatest interest. All other subjects, such as etiology, diagnosis, operation, and the like, are insignificant to the patient as compared with that of the permanent cure.

What constitutes a permanent cure? Authorities differ on this question. No one is willing to admit a less time than three years as decisive. Recently some surgeons have placed the limit of time as five years. In some of my cases ten to eighteen, and even twentyfive years have elapsed with no return of the disease.

The percentage of returns after three years is so insignificant that it would seem wise for surgeons to accept this standard, not only for the encouragement of the patient, which is no small fact in the management of the disease, but because it establishes a fixed standard from which the case from an operative as well as an optimistic point of view can be judged.

\section{ADVISABILITY OF OPERATION IN ADVANCED CASES}

There is much diversity of opinion on this question. From my own experience I feel certain that it is a wise course to operate unless the mediastinal glands are involved, or unless there is visceral metastasis, or the growth is adherent to the chest wall. I have been astonished at some results after operation, in cases in which I felt absolutely certain a return and death would follow in a few months. Yet these patients have recovered and have remained free from the disease for years, and thus have demonstrated the error of a too conservative view held by the profession at large.

In some cases in which the outlook was most unfavorable, as manifested by extensive ulceration, hemorrhage, wide-spread axillary involvement, the final results have heen entirely satisfactory. No better proof of this statement can be made than by a brief reference to three cases occurring in the list of thirty-nine cases of cancer of the breast. It seems pertinent to call attention to these three cases because they demonstrate the possibility of a cure when everything looked hopeless. I admit that we can not place much reliance on a cure when cases present such a chain of unfavorable symptoms. It only shows that surgeons should not withhold an operation from the patient, even when the outTonk is most discouraging. The patients should have the henefit of the doubt; it is always best, however, to explain to the friends the situation, and then they will not be too greatly disappointed if the operation is attended with a quickly fatal result.

The first patient had a foul, ulcerating, hemorrhagic, and fungous breast, with axillary enlargement. I advised the operation, not with the expectation of curing the disease, but purely from an humanitarian point of view. I felt sure that the hemorrhages conld in a measure be controlled, the offensive smell to a greater or Jess extent be removed, the constant pain, at least for a. while be relieved, and the patient be made more comcortable during the few months she lived. To my surprise alter nine years this patient has been free from the disease, and to all intents and purposes is at the present time in perfect health. The operation was radical as the circumstances permitted, and I am at loss to explain the cure under these unfavorable conditions.

The second patient was the victim of a fungons, hemorrhagic foul-smelling carcinoma of the breast. I hesitated to operate at all, but did so at the request of the husband, with a full understanding that the operation was one to palliate the distressing symptoms, anil with no expectation of cure. This operation was performed five years ago, and only recently the patient died from an acute disease, with no evidence of any return of the cancer.

Both of these tumors were examined by microscopists. of world-wide reputation, who pronounced them carcinoma. I merely mention these two cases in the list of thirty-nine to illustrate the fact that sometimes recovery takes place in the most hopeless cases. Such instances appear to me to urge the surgeon to operate in the most desperate cases, with the most forlorn prognosis, unless, as previously indicated, there is involvement of the mediastinal glands, or visceral metastasis. or adhesion of the growth to the chest wall. I can offer no explanation as to the behavior of these two tumors, but thev both seem worthy of record as evidence that our knowledge is still incomplete in regard to the course of certain types of cancer of the breast.

The third case is one in which I had operated nearly ten years ago and later a metastasis occurred in the lung with an extensive hemothorax. By aspiration a very large quantity of bloody fluid was withdrawn to relieve a severe dyspnea. To my utter astonishment, this patient, after the paracentesis, began immediately to improve from an almost bed-ridden condition, and for many years was in fairly good health and was in the habit of coming to my office from a distance for two or three vears. From a study of these cases, bearing on the subject of permanent cures, I have been impressed with the fact that the histologic formation of the tumor influences in a marked degree the question of permanent cures. For example, I have found that the tumors which show structures departing but slightly from the normal, correspond in nearly every case with the group of cases, the clinical histories of which were favorable, because in these cases no return of the carcinoma occurred. The tumors that showed a great departure from the normal structure occurred in the cases with unfavorable clinical histories. The more typical the structure, therefore, the more favorable the prognosis; the more atypical, the more unfavorable the prognosis. In other words, epithelioma develops by asexual cell proliferation; the more embryonic the cell is, the more malignant the growth; and the nearer the cell approaches the normal, the less malignant the growth.

\section{CONDITIONS REQUIRED FOR COMPLETE RECOVERY}

The early and radical operation is of paramount importance, since the facts I have already mentioned clearly demonstrate the value of this rule. The early operation is indicated because glandular infection is not likely to be present, and the patient is in better physical health, from absence of worry and pain. This fact should impress the family physician with the responsibility of referring his patient early to a surgeon, who then must assume the responsibility of this imporfant question. No growth in the breast, however small, should be disregarded, especially if the patient is ap- 
proaching the menopause. It is by a view to prophylaxis that the mortality of death from cancer of the breast is to be still further reduced.

The more radical the operation, within reasonable limits, the better the prognosis. It seems unnecessary and a loss of time to discuss this point, since it is universally admitted that the more radical the operation, the less likely it is that the disease will return. The difficulty seems to be as to what constitutes a radical operation. Surgeons agree that all skin over the infected area should be sacrificed. The breast, with Sir Astley Cooper's ligaments, should be entirely removed. the pectoral fascia and muscles should be excised, the axillary glands, the perimammary and retromammary fat, likewise the paramammary areolar tissue should be dissected out as the minimum operation. If the cancer involves the ribs, perforates the thoracic wall, and has already infected the mediastinal glands, no operation, however radical, would be attended with any success. The same rule may also apply to that form of cancer termed en cuirasse, or en plaques, or acute miliary carcinosis. I am firmly convinced that any radical operation, more than has been described, will be of no avail. The element of time should be seriously considered, since the performance of any operation which requires the patient to be under an anesthetic for four or five hours, is attended with a risk, which if possible should be obviated. These prolonged operations are sometimes followed by ether pneumonia, acute sepsis, profound shock, suppression of urine, and many other like conditions, which jeopardize the life of the patient. In other words, we must not.lose sight of some of the general principles connected with surgery, at the expense ff the elaboration of an operation, the performance of which eliminates some of the best precepts and practices of our art.

In most of my cases in which no return has ever occurred, even though twenty-five years have elapsed since the operation, the patients, almost. without exception, were operated on within six months from the first appearance of the disease. This point seems to me of the greatest importance in relation to the prognosis, and without question the earlier the operation the more certain it is that a permanent cure can be effected. At this point naturally the question arises as to a certainty of diagnosis within six months. I admit that it is often difficult to determine with absolute certainty that any small nodule in the breast is carcinomatous. The obstacle presented by the theory that a correct diagnosis of carcinoma within six months is a sine qua non for operative interference has been removed in my own mind, by a rule that all nodules or tumors in a breast in a woman at the time of or near to, or after the menopause, especially with a depressed nipple and skin dimpling, should be removed by a complete ablation of the breast. The risk run is too great if surgical interference is deferred until the diagnosis is made certain

In several cases during the past few years I have adopted this procedure, and to my surprise the microscopic report has been returned with the diagnosis of incipient epithelioma. In such cases the patients do well as they are taken early in the course of the disease, and the nodule is small and surrounded by healtly glandular tissue. In the hands of a good surgeon with aseptic environment the risk of removing the breast is so slight that it may almost be disregarded as a factor in determining the question of an operation.
A point still further to consider is that in a large majority of cases in which a nodule or tumor is felt, it is almost certain in time to become malignant, if it has not already begun to undergo malignant degeneration. This same law of early surgical interference holds good in case of tuberculous induration, in syphilitic ulceration, in actinomycosis, or in other infective processes.

The question is more difficult when the patient is a young woman, and the growth is adenomatous. Such cases are more difficult to manage; but I am still of the opinion that all adenomata in the young should be removed, and in the case of very young girls only the nodule itself excised and examined, and if then found to be undergoing epitheliomatous changes, the entire breast should be removed by a radical operation. In the case of young girls or young women, the breast can be raised by a flap with an incision under the breast and in its fold, and turned on the thoracic wall, and the adenoma attacked from the posterior surface of the gland. After excision of the adenoma the breast can be turned down and sutured with the scar completely hid from view. I have done this and preserved the symmetry of the breast; and I have record of cases of this kind in which the breast subsequently performed its function of lactation.

In cases of chronic mastitis in a woman over forty, if pain is a constant factor, and the interstitial induration is marked, the breast should be removed to prevent the possibility of malignant degenerative changes. Within a few weeks I removed a breast for painful mastitis, and Dr. Norris reports that beginning epithelioma is present.

\section{THE MANAGEMENT OF CASES THAT DO NOT ADMIT OPERATION}

These are the cases that tax the surgeon's skill, and cause severe suffering to the patient. Nothing is more distressing to the surgeon and painful to the patient than to watch the progress of a fatal disease with the full knowledge of the condition-both waiting, utterly helpless, until the end comes, and death closes the scene.

In such cases the surgeon's duty is to mitigate the suffering, and to make the patient as comfortable as possible with a view to euthanasia. The different methods of treatment of cancer of the breast, in cases in which no operation is possible, can be discussed very briefly. The $x$-ray is one of the methods now in vogue. One writer has reported a dozen cases of cancer of the breast in which cures were effected by the $x$-ray. The absence of a microscopic examination of any of the tumors, and the fact that not a single case in the list had yet reached the three years' limit of time make these cases valueless as a basis on which to study results.

The $x$-ray is a force that should not be employed by one who is not an expert in its use, because its employment even by the best men has been followed by violent dermatitis, by ulcers that fail to heal, and that finally became the starting point of epithelioma. In a recent article by Pusey, some interesting clinical facts have been brought out in connection with the $x$-ray, which are worthy of mention. He thinks that the $x$-ray is not suitable for cases of carcinoma in which the adjacent lymph nodes are involved. It is also futile in cancer of the neck, or of the viscera. In recurrent carcinoma of the breast, alleviation of suffering for a few months has followed the $x$-ray treatment, but no cures have 
followed a year's test. In primary carcinoma of the breast I fail to find an authentic case which has been permanently cured; but if for any reason no operation can be performed, the $x$-ray is worthy of trial on account of the possibility of alleviation of suffering.

Radium is a new therapeutic measure, the value of which is unknown. This is conceded even by those who have used it most extensively in the treatment of maligllant disease. It is a force that is most powerful, and the permanency of the cures claimed by its advocates is not yet determined.

It is only fair to say that I do not wish to place myself. on record as depreciating in any way the use of radiIIm, or any of these agencies. I only contend that they have their limited use in certain varieties of malignant ulcers, and that in no case should they take the place of surgical procedure, except in cases unsuitable for operation.

The important clinical fact must not be lost sight of, that in nearly all cases, like those of reported permanent cures by the $x$-ray, or those cases of reported cure by radiun, a microscopic examination is wanting to prove the diagnosis correct. In certain superficial epithelial ulcers radium has effected a cure.

The serum treatment of cancer of the breast has not yet yielded any satisfactory results. As far as I can ascertain from the best authorities, no cures have been accomplished by serum injection in cases of cancer of the breast.

In a recent communication, Dr. Clowes of the State Cancer Laboratory states that so far as he is aware "no results have ever been published from strictly scientific institutions regarding successful treatment of (ancer of the breast by means of serum. We have ourselves experimented in the past with horse serum, and the serum of dogs and goats, after the animals in question had been treated with repeated doses of cancer material.

"The results have been negative, although in one (ase the development of the tumor was unquestionably delayed for some time, but if anything grew more rapidly than would have otherwise been the case towards the end. Even were a case of successful treatment reported in the literature, in which pathologic examination excluded error of diagnosis, I should still feel inclined to consider it one of spontaneous recovery."

The serum treatment of cancer of the breast must still- be held subjualice. It has failed to cure cancer in any form, but it has succeeded in the hands of some surgeons in effecting a cure in certain varieties of sarcoma.

The surgeon is utterly powerless to treat cancer of the breast by other means than operation until our scientific laboratories discover whether cancer is a metabolic or a parasitic disease. When this great discovery has been made, the treatment of cases of cancer in which operation is not possible will in all probability be accomplished.

The emplorment of drugs for the relief of pain is indicated in cases of inoperable cancer of the breast, and these remedies should be used in sufficient doses to accomplish the object. The management of cases of cancer of the breast in which operation is not admissible should engage our study, and it is to be hoped that before long some remedy will be forthcoming that will cure this disease when it has advanced beyond the stage at which it is curable by operation.

542 Madison Avenue.

\section{CANCER OF THE BREAST}

WIJLIAM L. RODMAN, M.D., LL.D.

Professor or the Principles of Surgery and Clinical surgery, MedicoChirurgical College; Surgeon to Presbyterian, Jewish and Philadelphia General Hospitals

\section{PHILADELPHIA}

It is manifest that only a few of the more important and fairly well-demonstrated features of carcinoma can claim our consideration. It would be profitless to wander into the attractive yet elusive field of the etiology of carcinoma in general. While much has been written during the past year tending to show the parasitic origin of cancer, I am still of the opinion, as I have ever been, that the arguments of those who can see nothing in carcinoma but a germ disease are, to say the least, inconclusive. Attractive as this subject is and as much as I should like to discuss it, there are other features of cancer about which there is less ground for speculation and concerning which a discussion on this occasion is both more apposite and germane. We, as practitioners of medicine and surgery can safely leave this most important, yet unsolved, problem in the hands of laboratory experts, feeling hopeful if not entirely confident that convincing proof may ere long be forthcoming.

More will be gained at this time by insisting on that which is, and has long been, definitely known to operating surgeons, but is yet hardly appreciated by practitioners, who, as a rule, first see patients with mammary cancer and are consulted for advice. The fate of these unfortunate victims of cancer is largely in the keeping of the family physician, and he can not escape the responsibility; to show the importance of this fact is, I conceive it, the first duty incumbent on me. An early diagnosis, when the disease is strictly local, makes an operative cure not only possible, but highly probable, whereas a tardy recognition of mammary carcinoma can not be atoned for by the most extensive and brilliant operative procedure. Patients too long neglected are doomed to a lingering, loathsome and painful death simply because the disease is no longer local, but has become general. That the transition from a local, discrete and entirely operable condition, to a general, disseminated and inoperable one may take place quickly, is demonstrated by the most elementary anatomic and pathologic investigation. Cancer begins as an undue proliferation of the epithelial cells of the mamma and continues to be a strictly local neoplasm and therefore easily and completely removable if attacked before metastases have occurred in the neighboring or distant lymphatic glands and viscera. I shall show that early operation will cure 80 per cent. of all cases without glandular involvement, and also demonstrate as conclusively that infection of even the nearest lymphatic nodes-the axillary-permits an abiding result in only 25 per cent. of such cases. Need more be said to those who continue to hold pessimistic opinions, the offspring of the false teachings of a past era? If the profession could be made first, to realize, and second, to teach the laity that mammary growths are usually malignant and should be considered and treated as such until their benignity is proven, the operative results would be far better than they are. As it is, they are much better than is generally appreciated, and, notwithstanding the many inexcusable blunders in diagnosis, and the more frequent belated reference of such cases to surgeons, one-third of such patients at the very least are permanently cured. Until we can say that at least two. 